\title{
Soft-Lithographic Fabrication of Ni Nanodots Using Self-Assembled Surface Micelles
}

\author{
Young-Soo Seo ${ }^{1}$, Jungsoo Lee ${ }^{2}$, Kyungil Lee ${ }^{3}$, and Taewan Kim ${ }^{1 *}$ \\ ${ }^{1}$ Nano Advanced Materials Engineering, Sejong University, Seoul 143-747, Korea \\ ${ }^{2}$ Korea Atomic Energy Research Institute, Daejeon 305-600, Korea \\ ${ }^{3}$ Center for Spintronics Research, Korea Institute of Science \& Technology, Seoul 136-791, Korea
}

(Received 22 May 2008)

\begin{abstract}
This study proposes a simple nano-patterning process for the fabrication of magnetic nanodot arrays on a large area substrate. Ni nanodots were fabricated on a large area (4 inches in diameter) Si substrate using the soft lithographic technique using self-assembled surface micelles of Polystyrene-block-Poly(methyl methacrylate) (PS-b-PMMA) diblock copolymer formed at the air/water interface as a mask. The hexagonal array of micelles was successfully transferred to a Ni thin film on a Si substrate using the Langmuir-Blodgett technique. After ion-mill dry etching, a magnetic Ni nanodot array with a regular hexagon array structure was obtained. The $\mathrm{Ni}$ nanodot array showed in-plane easy axis magnetization and typical soft magnetic properties.
\end{abstract}

Keywords : Ni nanodot, self-assembly, nano-patterning process, diblock copolymer, surface micelle

\section{Introduction}

Nano-patterned magnetic dot arrays have drawn significant attention in recent years due to their potential applications in ultrahigh density information storage and magnetoelectronic devices and for basic research into magnetic domain structures [1]. Many nano-patterning processes, such as electron-beam, nano-imprint, interference, and self-assembled lithography, have been used to fabricate nanodot arrays. Among these processes, selfassembled lithography is perhaps the best candidate for simple and inexpensive nano-patterning on a large area substrate, but the key issue is how to control the size and array of nanodots.

We studied a self-assembled nano-patterning process using diblock copolymers consisting of hydrophobic and hydrophilic blocks in an effort to fabricate regular $\mathrm{Ni}$ nanodot arrays on a large area substrate. Diblock copolymers that consist of hydrophobic and hydrophilic blocks spread out at the air/water interface to spontaneously form self-assemblies called "surface micelles" [2-4]. The high water surface tension induces the spreading out of the hydrophilic blocks in order to decrease the surface free energy of the water, where the hydrophobic blocks are

*Corresponding author: Tel: +82-2-3408-3691

Fax: +82-2-3408-3664, e-mail: twkim@sejong.ac.kr aggregated to form a core surrounded by a corona of hydrophilic blocks. These surface micelles can be easily transferred onto substrates such as $\mathrm{Si}$ wafers from the water surface using the Langmuir-Blodgett technique. The pancake-like core of the surface micelle can be used as a mask [5], which offers a means of developing inexpensive lithographic techniques for the production of nano-scale devices. The system we chose to study was a Polystyrene-block-Poly(methyl methacrylate) (PS-b-PMMA) diblock copolymer, in order to prepare a 2-dimensional (2D) hexagonal array of surface micelles as a mask for fabricating Ni nanodot arrays on Si wafers.

\section{Experiments}

The symmetric diblock copolymer, PS-b-PMMA, (Molecular weight of 193,600-201,000 g/mol and polydispersity of 1.14) was dissolved in chloroform as a spreading solvent and the solution concentration was fixed at $1 \mathrm{mg} /$ $\mathrm{ml}$. The solution was spread on a Langmuir trough made by KSV instruments Ltd. with a maximum area of 704 $\mathrm{cm}^{2}$, using deionized water as the subphase. The subphase water temperature was maintained at $20^{\circ} \mathrm{C}$ by means of a thermostat circulator during the experiment. After the spreading, a 15 min waiting period was needed to allow the chloroform to completely evaporate before compressing the solution by means of a barrier. 
The Langmuir layer of the diblock copolymer was compressed with a constant barrier speed of $5 \mathrm{~mm} / \mathrm{min}$ until the target surface pressure was reached. A $25 \mathrm{~nm}$ thick Ni film on a 4 inch $\mathrm{Si}$ wafer prepared by sputtering was immersed in the water before the spreading, which was used for the Langmuir-Blodgett (LB) film transfer process. After reaching the target pressure, the $\mathrm{Ni} / \mathrm{Si}$ substrate immersed in the water was vertically pulled out from the water subphase at a constant speed of $2 \mathrm{~mm} / \mathrm{min}$, while keeping the surface tension constant. This process enables the surface micelle array to be transfered onto the substrate from the water surface without undergoing any structural changes. The topography of the pattern was measured by Atomic Force Microscopy (AFM) a Dimension 3000 scanning probe microscope. The LB film was then inserted into an Ar ion mill dry etcher with a base pressure of $3.7 \times 10^{-7}$ Torr. A 200 Watt RF plasma was used to sputter the film for 2.1 minutes at normal incidence. The etching condition was adjusted so that the etch rate of the Ni thin film was approximately twice as fast as those of PMMA and PS, with the average etch rate of $\sim 10 \mathrm{~nm} / \mathrm{min}$. The total etching time was $\sim 2.1 \mathrm{~min}$, which was determined by the optical end point detection of $\mathrm{Si}$, which indicated the exposure of the $\mathrm{Si}$ surface beneath the Ni layer. The sample was rinsed in toluene to remove any organic contaminants before charaterization by AFM and Scanning Electron Microscopy (SEM).

The magnetization measurement of the $\mathrm{Ni}$ nanodot array was performed with samples having a size of 14.07 $\mathrm{mm}^{2}$ up to a field of $5 \mathrm{kOe}$ using an Alternating Gradient Magnetometer (AGM).

\section{Results and Discussion}

As soon as the PS-b-PMMA diblock copolymer solution in chloroform spreads out on the water, PS-b-PMMA starts to aggregate, while the chloroform evaporates. The hydrophobic PS blocks aggregate into a hard core, while the relatively hydrophilic PMMA blocks spread out to form a surface micelle in the form of a corona on the water. The interaction between the surface micelles is repulsive, because the PMMA coronas repel each other on the water. Therefore, the surface micelle forms 2dimensional (2D) hexagonal arrays, as shown in Fig. 1.

After spreading out, as the surface area of the trough is decreased by the squeezing of the barrier, the surface pressure keeps increasing until the film is physically deformed at pressures above $50 \mathrm{dyne} / \mathrm{cm}$. The characteristic surface pressure-area $(\pi-A)$ isotherm is shown in Fig. 2. The isotherm shows two distinct regions which are separated by the "shoulder" indicated by the asterisk in

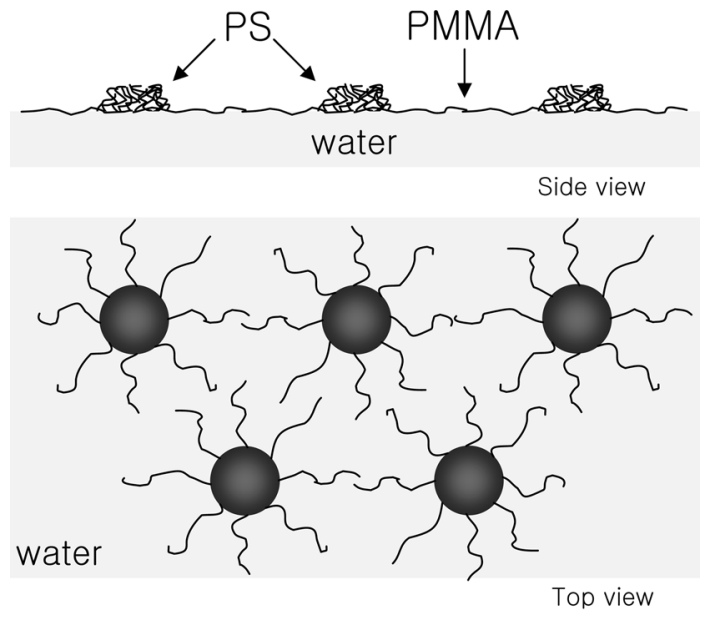

Fig. 1. Schematic view of the self-assembled structure of the Polystyrene-block-Poly(methyl methacrylate) (PS-b-PMMA) diblock copolymer, called a surface micelle. The hydrophobic PS blocks aggregate into a nano-sized core, while the relatively hydrophilic PMMA blocks form a surface micelle in the form of a corona at the air/water interface (side view). The surface micelle array is hexagonally packed due to the repulsive interaction between the coronas (top view).

the isotherm, where the type of interaction between the surface micelles changes. Before the shoulder, the surface micelles form a hexagonal packing array without disturbing the circular shaped conformation of the corona chains. After the shoulder, on the other hand, the area occupied by the surface micelle becomes smaller and, therefore, the circular shape of the coronal chains is squeezed and distorted.

Three samples were obtained at three different surface pressures of 10,30 , and $50 \mathrm{dyne} / \mathrm{cm}$, respectively, as indicated by the arrow in Fig. 2. The corresponding surface micelle structure imaged using AFM after being transferred onto a $\mathrm{Si}$ wafer is also displayed in the inset. At a surface pressure of $10 \mathrm{dyne} / \mathrm{cm}$, just before the shoulder, the surface micelle arrays are hexagonally packed, as represented by the hexagon in the Figure. Each surface micelle is shown to have a uniform size hexagonal array of surface micelles with a PS core $\sim 13 \mathrm{~nm}$ in height and $\sim 100 \mathrm{~nm}$ in diameter with a center to center distance of $\sim 250 \mathrm{~nm}$ and a stretched PMMA corona which is approximately $1 \mathrm{~nm}$ thick. At a surface pressure of $30 \mathrm{dyne} / \mathrm{cm}$, the array structure loses its ordering and becomes distorted. At a surface pressure of 50 dyne $/ \mathrm{cm}$, just before the collapse of the film, the arrays are highly squeezed and appear to be aligned in arbitrary directions.

We transferred the surface micelle array onto a 4 inch $\mathrm{Ni} / \mathrm{Si}$ substrate at 10 dyne/cm and then subjected it to Ar ion mill dry etching. A schematic drawing of the ion mill 


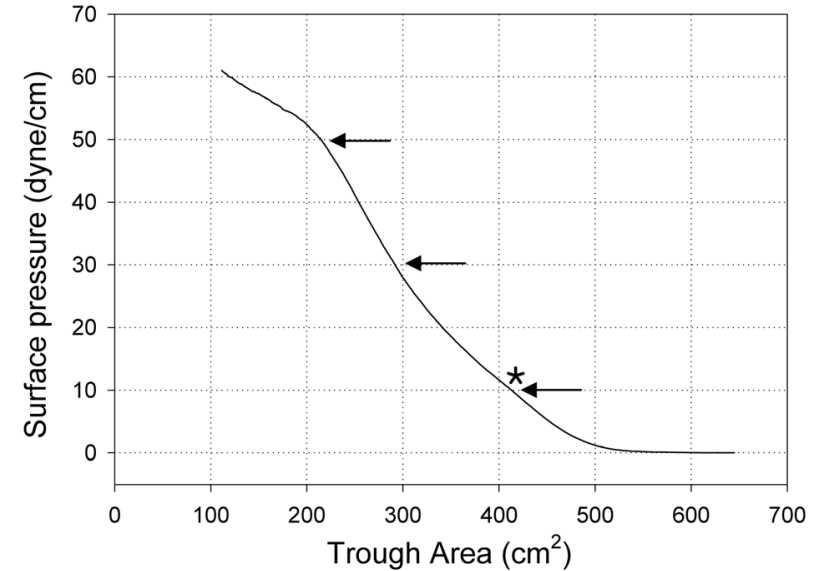

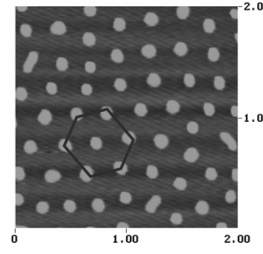

10 dyne/cm

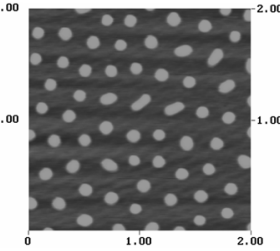

30 dyne/cm

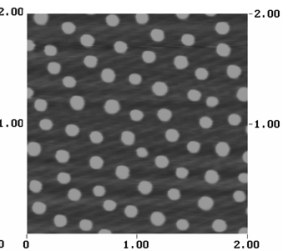

50 dyne/cm
Fig. 2. The surface pressure-area $(\pi-A)$ isotherm of the PS-bPMMA diblock copolymer is displayed. The arrows indicate where the surface micelle is transferred onto the $\mathrm{Ni} / \mathrm{Si}$ substrate. The asterisk marker indicates the "shoulder". Three AFM images of the surface micelles transferred at 10, 20, and 30 dyne/cm, respectively, are presented.

dry etching process is presented in the left inset of Fig. 3. After the ion milling, the topography of the pattern was measured using AFM, which shows that the height and diameter of the Ni dots, viz. $\sim 14 \mathrm{~nm}$ and $\sim 120 \mathrm{~nm}$, respectively, are only slightly greater than those of the PS cores and that the hexagonal array is unchanged after the ion milling, as shown in the right inset of Fig. 3. From the SEM image of the Ni pattern in Fig. 3, it is observed that the dots appear to be much brighter than the surrounding native Si oxide background, which is consistent with the larger electron density of $\mathrm{Ni}$. In the figure, the dot sizes are shown to be regular for the circular micelles, although dumbbell shaped micelles appear occasionally. We think that the dumbell shape of the micelles results from the fact that the evaporation rate of the spreading solution was not uniform during the spreading procedure. It is noted that the hexagonal array of $\mathrm{Ni}$ dots was measured on all parts of the 4 inch $\mathrm{Si}$ wafer. We confirm that the pancake-like PS core of the surface micelle effectively protected the $\mathrm{Ni}$ film from the ion milling so as to generate Ni nanodots.

The in-plane and out-of-plane magnetization hysteresis loops of the Ni nanodot array are shown in Fig. 4. Com-

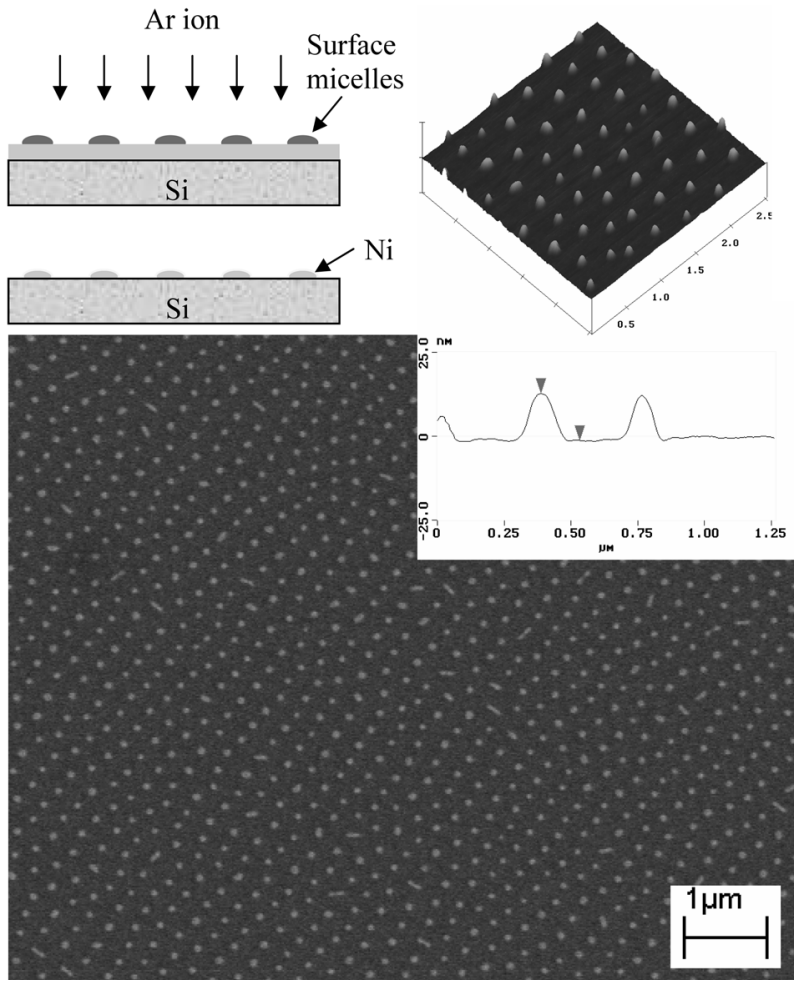

Fig. 3. A schematic drawing of Ar ion milling on the surface micelle array is presented in the left inset, where the Ni under the PS core remained unsputtered, while the Ni under the PMMA corona was removed first. The SEM image of the Ni pattern is displayed and the topography of Ni observed using AFM is also shown in the right inset.

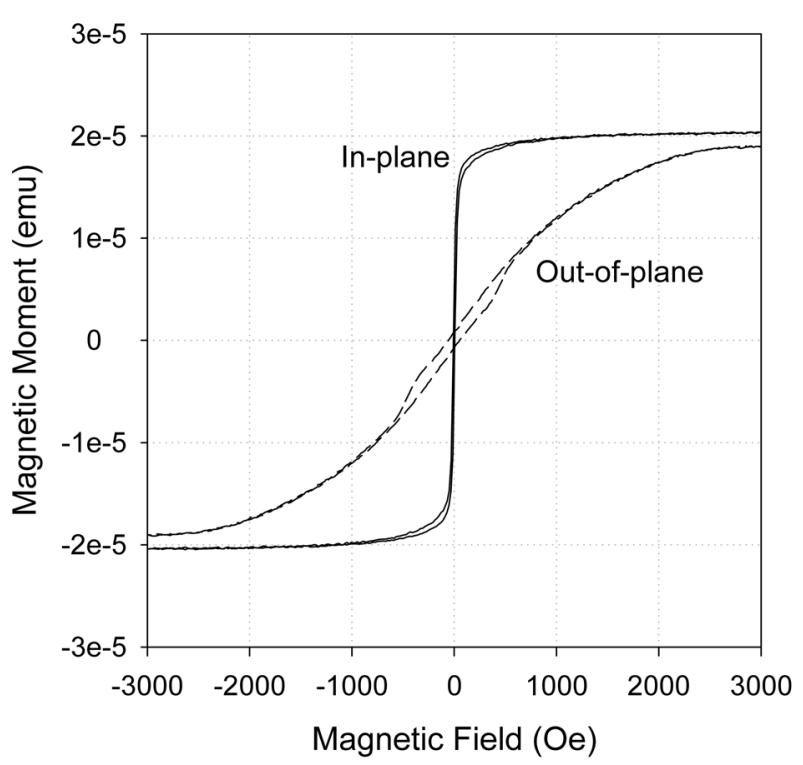

Fig. 4. The in-plane and out-of-plane magnetic hysteresis loops of the Ni nanodot array (Sample size: $14.07 \mathrm{~mm}^{2}$ ).

pared to the out-of-plane magnetization curve measured with the applied field perpendicular to the $\mathrm{Ni}$ nanodot 
plane, the in-plane magnetization curve measured with the applied field parallel to the $\mathrm{Ni}$ nanodot plane was more easily saturated, which means that the array has an in-plane easy axis of magnetization, which is to be expected given the pancake shape of the Ni nanodots with their smooth slope. Considering the fraction of the $\mathrm{Ni}$ nanodot area to the total area and the bulk Ni saturation magnetization of $484 \mathrm{emu} / \mathrm{cm}^{3}$ [6], the expected magnetic moment of the Ni nanodot array for the sample size of $14.07 \mathrm{~mm}^{2}$, which was used for the AGM measurement, was estimated. The magnetic moment of the $\mathrm{Ni}$ nanodot array was $1.72 \times 10^{-5} \mathrm{emu}$, which is close to the value of $2 \times 10^{-5} \mathrm{emu}$ obtained from the total volume of the $\mathrm{Ni}$ nanodots in the sample, which indicates that the Ni nanodots are almost pure Ni. The magnetization hysteresis loop of the Ni nanodot array showed soft magnetic properties, with a coercivity of $\sim 8$ Oe and reduced remanance (M/ $\mathrm{M}_{\mathrm{s}}$ ) of 0.2 .

\section{Conclusion}

The symmetric diblock copolymer, PS-b-PMMA, was self-assembled into a hexagonal array of surface micelles at the air/water interface. The surface micelle comprises a core of PS blocks and a corona of PMMA blocks. The array was successfully transferred onto the $\mathrm{Ni} / \mathrm{Si}$ substrate at a surface pressure of $10 \mathrm{dyne} / \mathrm{cm}$ and used as a mask to produce a Ni nanodot array, in which the $\mathrm{Ni}$ nanodots have a pancake shape with a smooth slope. On the basis of the calculated and measured magnetic moments, the $\mathrm{Ni}$ nanodots were found to be almost pure Ni. The magnetization hysteresis loop of the Ni nanodot array showed soft magnetic properties with a coercivity of $\sim 8 \mathrm{Oe}$.

\section{Acknowledgement}

This work was supported by the faculty research fund of Sejong University in 2006.

\section{References}

[1] J. Chang, B. A. Gribkov, H. Kim, H. Koo, S. Han, V. L. Mironov, and A. A. Fraerman, J. Magnetics, 12(1), 17 (2007).

[2] B. Lin, S. A. Rice and D. A. Weitz, J. Chem. Phys., 99, 8308 (1993).

[3] Z. Li et al. Langmuir 11, 4785 (1995).

[4] K. Juliet et al. Langmuir 15, 7714 (1999).

[5] M. V. Meli, A. Badia, P. Grutter, and R. B. Lennox, Nano Letters 2, 131 (2002).

[6] B. D. Cullity, Introduction to Magnetic Materials, Addison-Wesley Publishing Company, New York, p. 617 (1972). 